\title{
ON THE SECOND ORDER EQUATIONS WITH NONLINEAR IMPULSES. FREDHOLM ALTERNATIVE TYPE RESULTS
}

\author{
Pavel Drábek - Martina Langerová
}

\begin{abstract}
We consider the semilinear homogeneous Dirichlet boundary value problem for the second order equation on a finite interval with nonlinear impulses in the derivative at prescribed points. We introduce Landesman-Lazer type necessary and sufficient conditions for resonance problems and generalize the Fredholm alternative results for linear operators. An interaction between nonlinear restoring force and nonlinear impulses is presented.
\end{abstract}

\section{Introduction}

In this paper we study the second order Dirichlet boundary value problem with nonlinear impulse effects in the first derivative of the solution. Similar problems were considered in papers [1], [7], [13] and [9], for periodic problems see e.g. [12]. The practical importance of models the solutions of which include instantaneous impulses depending on the position that result in jump discontinuities in velocity, but with no change in position, was stressed in papers [2], [3], $[8]$ and $[10]$.

2010 Mathematics Subject Classification. Primary 34A37, 34B37; Secondary 34F15, $47 \mathrm{H} 11$.

Key words and phrases. Ordinary differential equations of the second order with impulses, resonance problems, Landesman-Lazer conditions.

This research was supported by the Grant 13-00863S of the Grant Agency of Czech Republic and by the European Regional Development Fund (ERDF), project "NTIS New Technologies for the Information Society", European Centre of Excellence, CZ.1.05/1.1.00/02.0090. 
We consider nonlinear impulses at prescribed points and study their influence on the solvability of the Dirichlet problem. In particular, we concentrate on the so called resonance problems and give necessary and sufficient conditions for the existence of a solution.

Let us consider the linear Dirichlet boundary value problem

$$
\begin{aligned}
-u^{\prime \prime}(x)-\lambda u(x) & =f(x), \quad \text { for a.e. } x \in(0, \pi), \\
u(0)=u(\pi) & =0,
\end{aligned}
$$

where $\lambda \in \mathbb{R}$ is a spectral parameter and $f \in L^{2}(0, \pi)$.

Let $0=t_{0}<t_{1}<\ldots<t_{p}<t_{p+1}=\pi$ be given points and $I_{j}: \mathbb{R} \rightarrow \mathbb{R}$, $j=1, \ldots, p$, be given continuous functions. We are interested in the solutions of (1.1) satisfying the impulse conditions in the derivative:

$$
\Delta u^{\prime}\left(t_{j}\right):=u^{\prime}\left(t_{j}{ }^{+}\right)-u^{\prime}\left(t_{j}{ }^{-}\right)=I_{j}\left(u\left(t_{j}\right)\right), \quad j=1, \ldots, p .
$$

Assume that $\lambda \neq n^{2}, n \in \mathbb{N}$, i.e. $\lambda$ is not an eigenvalue of the homogeneous problem $(f \equiv 0$ in $(1.1))$, and all $I_{j}$ have sublinear growth at $\pm \infty$ :

$$
\lim _{|s| \rightarrow \infty} \frac{I_{j}(s)}{s}=0, \quad j=1, \ldots, p .
$$

Under these assumptions the problem (1.1) has a solution satisfying (1.2) for arbitrary $f \in L^{2}(0, \pi)$, see Theorem 1. We call (1.1), (1.2) a nonresonance problem.

Let $\lambda=n^{2}$ for some $n \in \mathbb{N}$. Then $\lambda$ is an eigenvalue of the homogeneous problem and $\phi_{n}(x)=\sin n x$ is the corresponding eigenfunction. In order to formulate the result we need to distinguish among the points $t_{j}, j=1, \ldots, p$, which belong to subintervals of $(0, \pi)$ where $\sin n x$ is positive or negative and also those which coincide with zero points of $\sin n x$. For this purpose let us denote

$$
\mathcal{I}_{+}=\left(0, \frac{\pi}{n}\right) \cup\left(\frac{2 \pi}{n}, \frac{3 \pi}{n}\right) \cup \ldots, \quad \mathcal{I}_{-}=\left(\frac{\pi}{n}, \frac{2 \pi}{n}\right) \cup\left(\frac{3 \pi}{n}, \frac{4 \pi}{n}\right) \cup \ldots
$$

Clearly, $\sin n x>0, x \in \mathcal{I}_{+}, \sin n x<0, x \in \mathcal{I}_{-}$. We arrange $t_{j}, j=1, \ldots, p$, into 3 sequences: $0<\tau_{1}<\ldots<\tau_{p_{+}}<\pi, \tau_{i} \in \mathcal{I}_{+}, i=1, \ldots, p_{+} ; 0<\sigma_{1}<$ $\ldots<\sigma_{p_{-}}<\pi, \sigma_{j} \in \mathcal{I}_{-}, j=1, \ldots, p_{-} ; \xi_{k} \in\{\pi / n, 2 \pi / n, \ldots,(n-1) \pi / n\}$, $k=1, \ldots, p_{0}$. Obviously, we have $p_{+}+p_{-}+p_{0}=p$ and $p_{0} \leq n-1$. At first we assume $p_{+}+p_{-}>0$. The impulse condition (1.2) is then written in the following form:

$$
\begin{aligned}
\Delta u^{\prime}\left(\tau_{i}\right)=I_{i}^{\tau}\left(u\left(\tau_{i}\right)\right), & i=1, \ldots, p_{+}, \\
\Delta u^{\prime}\left(\sigma_{j}\right)=I_{j}^{\sigma}\left(u\left(\sigma_{j}\right)\right), & j=1, \ldots, p_{-}, \\
\Delta u^{\prime}\left(\xi_{k}\right)=I_{k}^{\xi}\left(u\left(\xi_{k}\right)\right), & k=1, \ldots, p_{0} .
\end{aligned}
$$


We assume that $I_{i}^{\tau}, I_{j}^{\sigma}, I_{k}^{\xi}: \mathbb{R} \rightarrow \mathbb{R}, i=1, \ldots, p_{+} ; j=1, \ldots, p_{-} ; k=1, \ldots, p_{0}$, are continuous and sublinear functions (i.e. satisfy (1.3)). There exist limits $\lim _{s \rightarrow \pm \infty} I_{i}^{\tau}(s)=I_{i}^{\tau}( \pm \infty), \lim _{s \rightarrow \pm \infty} I_{j}^{\sigma}(s)=I_{j}^{\sigma}( \pm \infty)$ and the following inequalities hold:

$$
I_{i}^{\tau}(-\infty)<I_{i}^{\tau}(s)<I_{i}^{\tau}(+\infty), \quad I_{j}^{\sigma}(-\infty)<I_{j}^{\sigma}(s)<I_{j}^{\sigma}(+\infty)
$$

for all $s \in \mathbb{R}$ and $i=1, \ldots, p_{+} ; j=1, \ldots, p_{-}$. Note that infinite limits are allowed in (1.5).

Under these assumptions the problem (1.1) has a solution satisfying (1.4) if and only if the function $f \in L^{2}(0, \pi)$ is such that

$$
\begin{aligned}
\sum_{i=1}^{p_{+}} I_{i}^{\tau}(-\infty) \sin n \tau_{i}+\sum_{j=1}^{p_{-}} I_{j}^{\sigma}(+\infty) \sin n \sigma_{j}<\int_{0}^{\pi} f(x) \sin n x d x \\
<\sum_{i=1}^{p_{+}} I_{i}^{\tau}(+\infty) \sin n \tau_{i}+\sum_{j=1}^{p_{-}} I_{j}^{\sigma}(-\infty) \sin n \sigma_{j}
\end{aligned}
$$

see Theorem 4.1 .

In case $p_{+}+p_{-}=0$ the impulses are prescribed only at zero points of the eigenfunction $\sin n x$ and the problem (1.1) has a solution satisfying (1.4) if and only if the function $f \in L^{2}(0, \pi)$ satisfies the condition

$$
\int_{0}^{\pi} f(x) \sin n x d x=0
$$

see Theorem 4.2. The sublinearity of impulses can be relaxed and $I_{k}^{\xi}, k=$ $1, \ldots, p_{0}$, are supposed to be arbitrary continuous functions. Necessary and sufficient conditions for solvability of (1.1) and (1.1), (1.4) thus coincide.

Problem (1.1) with $\lambda=n^{2}, n \in \mathbb{N}$, is refered to as a resonance problem. The condition (1.6) is then called Landesman-Lazer type condition. Similar condition was introduced in paper [6] at the beginning of seventies and had been generalized in hundreds of subsequent papers in the following decades.

We also note here that if the inequalities in (1.5) hold in reversed order, we switch the role of $+\infty$ and $-\infty$ in (1.6) and similar result holds.

We illustrate our results on the following examples.

EXAMPLE 1.1. Let $\lambda=1$ in (1.1) and the impulse condition is of the form

$$
\Delta u^{\prime}\left(\frac{\pi}{2}\right)=\arctan u\left(\frac{\pi}{2}\right) \quad \text { or } \quad \Delta u^{\prime}\left(\frac{\pi}{2}\right)=-\arctan u\left(\frac{\pi}{2}\right) .
$$

Then (1.1) has a solution satisfying (1.7) if and only if $f \in L^{2}(0, \pi)$ and

$$
-\frac{\pi}{2}<\int_{0}^{\pi} f(x) \sin x d x<\frac{\pi}{2}
$$


EXAMPLE 1.2. Let $\lambda=4$ in (1.1) and the impulse condition is of the form

$$
\Delta u^{\prime}\left(t_{i}\right)=\arctan u\left(t_{i}\right), \quad t_{1}=\frac{\pi}{4}, t_{2}=\frac{\pi}{2}, t_{3}=\frac{3 \pi}{4} .
$$

In our notation $t_{1}=\tau_{1}, t_{2}=\xi_{1}, t_{3}=\sigma_{1}$. Then (1.1) has a solution satisfying (1.9) if and only if $f \in L^{2}(0, \pi)$ and

$$
-\pi<\int_{0}^{\pi} f(x) \sin 2 x d x<\pi
$$

EXAMPLE 1.3. Let $\lambda=4$ in (1.1) and the impulse condition is given by

$$
\Delta u^{\prime}\left(\frac{\pi}{2}\right)=u^{3}\left(\frac{\pi}{2}\right) .
$$

Then (1.1) has a solution satisfying (1.11) if and only if $f \in L^{2}(0, \pi)$ and

$$
\int_{0}^{\pi} f(x) \sin 2 x d x=0
$$

The reader certainly noticed that the Dirichlet problem (1.1) is linear and the nonlinearity has been involved just in impulses so far. Let $g: \mathbb{R} \rightarrow \mathbb{R}$ be a continuous function with sublinear growth, i.e. $\lim _{s \rightarrow \pm \infty} g(s) / s=0$, the limits $g( \pm \infty):=\lim _{s \rightarrow \pm \infty} g(s)$ exist and for all $s \in \mathbb{R}$

$$
g(-\infty)<g(s)<g(+\infty)
$$

Note that infinite limits are allowed in (1.13).

Consider nonlinear perturbation of (1.1):

$$
\begin{gathered}
-u^{\prime \prime}(x)-\lambda u(x)+g(u(x))=f(x), \quad x \in(0, \pi), \\
u(0)=u(\pi)=0 .
\end{gathered}
$$

Under above assumption on $g$, problem (1.14) with $\lambda \neq n^{2}, n \in \mathbb{N}$, has a solution for any $f \in L^{2}(0, \pi)$. On the other hand, problem (1.3) with $\lambda=n^{2}$ for some $n \in \mathbb{N}$ has a solution for $f \in L^{2}(0, \pi)$ if and only if

$$
\begin{array}{r}
g(-\infty) \int_{0}^{\pi}(\sin n x)^{+} d x-g(+\infty) \int_{0}^{\pi}(\sin n x)^{-} d x<\int_{0}^{\pi} f(x) \sin n x d x \\
<g(+\infty) \int_{0}^{\pi}(\sin n x)^{+} d x-g(-\infty) \int_{0}^{\pi}(\sin n x)^{-} d x
\end{array}
$$

see [4, Section 1.1] for details.

Our approach allows to combine the effects of nonlinear restoring force given by $g$ with the presence of nonlinear impulses at prescribed points, see (1.4). In the nonresonance case, i.e. $\lambda \neq n^{2}, n \in \mathbb{N}$, problem (1.14) with impulses (1.2) has a solution for arbitrary $f \in L^{2}(0, \pi)$, see Theorem 5.1. On the other hand, 
in the resonance case, i.e. $\lambda=n^{2}$ for some $n \in \mathbb{N}$, (1.14), (1.4) has a solution for $f \in L^{2}(0, \pi)$ if and only if

$$
\begin{aligned}
& g(-\infty) \int_{0}^{\pi}(\sin n x)^{+} d x-g(+\infty) \int_{0}^{\pi}(\sin n x)^{-} d x+\sum_{i=1}^{p_{+}} I_{i}^{\tau}(-\infty) \sin n \tau_{i} \\
& +\sum_{j=1}^{p_{-}} I_{j}^{\sigma}(+\infty) \sin n \sigma_{j}<\int_{0}^{\pi} f(x) \sin n x d x<g(+\infty) \int_{0}^{\pi}(\sin n x)^{+} d x \\
& -g(-\infty) \int_{0}^{\pi}(\sin n x)^{-} d x+\sum_{i=1}^{p_{+}} I_{i}^{\tau}(+\infty) \sin n \tau_{i}+\sum_{j=1}^{p_{-}} I_{j}^{\sigma}(-\infty) \sin n \sigma_{j}
\end{aligned}
$$

see Theorem 5.2.

If reversed inequalities hold in (1.13), i.e. $g(+\infty)<g(s)<g(-\infty)$ for all $s \in \mathbb{R}$, similar condition to (1.16) holds with the role of $+\infty$ and $-\infty$ mutually switched.

EXAMPLE 1.4. Let us consider problem

$$
\begin{aligned}
-u^{\prime \prime}(x)-u(x)-\arctan u(x) & =f(x), \quad x \in(0, \pi), \\
u(0)=u(\pi) & =0 .
\end{aligned}
$$

with impulses given by

$$
\Delta u^{\prime}\left(\frac{\pi}{2}\right)=\arctan u\left(\frac{\pi}{2}\right)
$$

Then (1.17), (1.18) has a solution for $f \in L^{2}(0, \pi)$ if and only if

$$
-\frac{3 \pi}{2}<\int_{0}^{\pi} f(x) \sin x d x<\frac{3 \pi}{2} .
$$

One can consider different situations depending on the values of $g( \pm \infty)$, $I_{i}^{\tau}( \pm \infty), I_{j}^{\sigma}( \pm \infty)$. The mutual connections among these values determine the range for $f \in L^{2}(0, \pi)$, given by the inequalities (1.16), for which the problem (1.14), (1.4) has a solution.

As far as we know, the second order problems with impulses of nonresonance type were considered in paper [11] but resonance problems have not been treated in the literature yet. Our results can be formulated and proved also for other types of boundary conditions like the Neumann or the periodic ones.

The reader should notice that conditions (1.6) and (1.16) may be regarded as a generalization of the condition $\int_{0}^{\pi} f(x) \sin n x d x=0$. From this point of view our results can be understood as a generalization of the Fredholm alternative for semilinear equations with nonlinear impulses. 


\section{Functional framework}

We start with precise definition of the notion of the solution of (1.1), (1.2). Following the ideas of [9], we say that $u$ is a classical solution of (1.1), (1.2) if the following conditions are fulfilled:

- $\left.u\right|_{\left(t_{j}, t_{j+1}\right)} \in H^{2}\left(t_{j}, t_{j+1}\right), j=0, \ldots, p$;

- the equation in (1.1) holds a.e. in $(0, \pi)$ and $u(0)=u(\pi)=0$;

- one sided limits $u^{\prime}\left(t_{j}{ }^{-}\right), u^{\prime}\left(t_{j}{ }^{+}\right)$exist and (1.2) holds for $j=1, \ldots, p$.

We say that $u \in H_{0}^{1}(0, \pi)$ (the usual Sobolev space) is a weak solution of (1.1), (1.2) if the integral identity

$$
\begin{aligned}
\int_{0}^{\pi} u^{\prime}(x) v^{\prime}(x) d x-\lambda \int_{0}^{\pi} u(x) & v(x) d x \\
= & -\sum_{j=1}^{p} I_{j}\left(u\left(t_{j}\right)\right) v\left(t_{j}\right)+\int_{0}^{\pi} f(x) v(x) d x
\end{aligned}
$$

holds for any test function $v \in H_{0}^{1}(0, \pi)$.

Note that the definitions of classical and weak solutions of (1.1), (1.4) follow with obvious modifications.

We refer to Lemma 3.1 and [9, Section 4] to claim that every weak solution of (1.1), (1.2) (or (1.1), (1.4)) is also a classical solution and vice versa. With this result in hands, we can look for (classical) solutions as for solutions of certain operator equation induced by (2.1).

Namely, we set $H:=H_{0}^{1}(0, \pi)$ with the scalar product

$$
(u, v)=\int_{0}^{\pi} u^{\prime}(x) v^{\prime}(x) d x
$$

and the norm $\|u\|=\sqrt{(u, u)}$, and introduce the operators $J, S, I^{*}: H \rightarrow H$ and an element $f^{*} \in H$ as follows:

$$
\begin{aligned}
(J u, v) & =\int_{0}^{\pi} u^{\prime}(x) v^{\prime}(x) d x, & (S u, v) & =\int_{0}^{\pi} u(x) v(x) d x, \\
\left(f^{*}, v\right) & =\int_{0}^{\pi} f(x) v(x) d x, & \left(I^{*}(u), v\right) & =\sum_{j=1}^{p} I_{j}\left(u\left(t_{j}\right)\right) v\left(t_{j}\right), \quad u, v \in H .
\end{aligned}
$$

The problem with nonlinear impulses (1.1), (1.2) is then equivalent to the operator equation

$$
J u-\lambda S u+I^{*}(u)=f^{*} .
$$

It follows easily from the definition of $J, S, I^{*}$ and from the compact embedding $H \hookrightarrow C[0, \pi]$ that $J$ is just identity, $S$ is linear compact operator and $I^{*}$ is (nonlinear) compact operator. Since $f \in L^{2}(0, \pi)$, an element $f^{*} \in H$ is well defined, too. 
To find a solution of (2.2) we use the Leray-Schauder Degree (LSD for short) argument (see [5] for details).

\section{Nonresonance case}

We start with nonresonance case and prove

Theorem 3.1. Let $\lambda \neq n^{2}, n \in \mathbb{N}, I_{j}: \mathbb{R} \rightarrow \mathbb{R}, j=1, \ldots, p$, be continuous sublinear functions (see (1.3)). Then (1.1), (1.2) has a solution for arbitrary $f \in L^{2}(0, \pi)$.

Proof. The properties of $J, S, I^{*}$ and $f^{*}$ imply that the LSD:

$$
\operatorname{deg}\left(J-\lambda S+I^{*}-f^{*} ; B_{R}, 0\right)
$$

where $B_{R}:=\{u \in H:\|u\|<R\}$, is well-defined if

$$
J u-\lambda S u+I^{*}(u)-f^{*} \neq 0
$$

for all $\|u\|=R$. If we find $R>0$ (possibly large) such that

$$
\operatorname{deg}\left(J-\lambda S+I^{*}-f^{*} ; B_{R}, 0\right) \neq 0,
$$

then there exists $u \in B_{R}$ which is a solution of (2.2). In order to find $R>0$ such that (3.1) holds, we use the homotopy invariance property of the LSD. To this end we introduce the homotopy

$$
\mathcal{H}(\kappa, u):=J u-\lambda S u-(1-\kappa) \delta S u+\kappa I^{*}(u)-\kappa f^{*}
$$

where $\kappa \in[0,1]$ is a homotopy parameter and $\delta>0$ is so small that $\lambda+(1-\kappa) \delta \neq$ $n^{2}, n \in \mathbb{N}$, for any $\kappa \in[0,1]$. We prove that there exists $R>0$ such that for all $u \in H,\|u\|=R$, and all $\kappa \in[0,1]$ we have

$$
\mathcal{H}(\kappa, u) \neq 0 .
$$

Assume for a while that (3.2) holds. We then conclude

$$
\begin{aligned}
\operatorname{deg}\left(J-\lambda S+I^{*}-f^{*} ; B_{R}, 0\right) & =\operatorname{deg}\left(\mathcal{H}(1, \cdot) ; B_{R}, 0\right) \\
=\operatorname{deg}\left(\mathcal{H}(0, \cdot) ; B_{R}, 0\right) & =\operatorname{deg}\left(J-(\lambda+\delta) S ; B_{R}, 0\right) .
\end{aligned}
$$

Since $J$ and $S$ are odd operators, by Borsuk Theorem (see [5, Chapter 5]), the last degree is equal to an odd number. In particular,

$$
\operatorname{deg}\left(J-(\lambda+\delta) S ; B_{R}, 0\right) \neq 0 .
$$

Hence (3.1) holds and the existence result follows.

It remains to prove (3.2). We perform the proof via contradiction. Assume that there exist $u_{m} \in H,\left\|u_{m}\right\| \rightarrow \infty, \kappa_{m} \in[0,1]$ such that $\mathcal{H}\left(\kappa_{m}, u_{m}\right)=0$. This is equivalent to

$$
J v_{m}-\lambda S v_{m}-\left(1-\kappa_{m}\right) \delta S v_{m}+\kappa_{m} \frac{I^{*}\left(u_{m}\right)}{\left\|u_{m}\right\|}-\kappa_{m} \frac{f^{*}}{\left\|u_{m}\right\|}=0
$$


where we set $v_{m}=u_{m} /\left\|u_{m}\right\|$. The last two terms tend to zero due to (1.3) and the fact that $f^{*}$ is a fixed element. Passing to subsequences if necessary, we may assume $v_{m} \rightarrow v$ (weakly) in $H$ for some $v \in H$ and $\kappa_{m} \rightarrow \kappa \in[0,1]$. Since $S$ is compact, $S v_{m} \rightarrow S v$ (strongly) in $H$. Now the strong convergence $J v_{m} \rightarrow J v$ follows from (3.5). In particular, $\|v\|=1$ and

$$
J v-(\lambda+(1-\kappa) \delta) S v=0 .
$$

Since $\lambda+(1-\kappa) \delta \neq n^{2}, n \in \mathbb{N}$, the above equation has only trivial solution, a contradiction.

\section{Resonance case}

In this section we deal with the resonance case. Assume that $\tau_{i}, \sigma_{j}, \xi_{k}$, $I_{i}^{\tau}, I_{j}^{\sigma}, I_{k}^{\xi}$ are as in Section 1. We have the following necessary and sufficient condition.

Theorem 4.1. Let $\lambda=n^{2}$ for some $n \in \mathbb{N}$. Then (1.1), (1.4) has a solution if and only if $f \in L^{2}(0, \pi)$ satisfies (1.6).

Proof. 1. Necessity of (1.6). Let $u$ be a solution of (1.1), (1.4). Then the integral identity

$$
\begin{aligned}
\int_{0}^{\pi} u^{\prime}(x) v^{\prime}(x) d x-n^{2} \int_{0}^{\pi} u(x) v(x) d x=-\sum_{i=1}^{p_{+}} I_{i}^{\tau}\left(u\left(\tau_{i}\right)\right) v\left(\tau_{i}\right) \\
-\sum_{j=1}^{p_{-}} I_{j}^{\sigma}\left(u\left(\sigma_{j}\right)\right) v\left(\sigma_{j}\right)-\sum_{k=1}^{p_{0}} I_{k}^{\xi}\left(u\left(\xi_{k}\right)\right) v\left(\xi_{k}\right)+\int_{0}^{\pi} f(x) v(x) d x
\end{aligned}
$$

holds for any $v \in H$. Take $v(x)=\sin n x$, an eigenfunction associated with $n^{2}$, in (4.1). The left-hand side as well as the third sum vanish and we get

$$
\sum_{i=1}^{p_{+}} I_{i}^{\tau}\left(u\left(\tau_{i}\right)\right) v\left(\tau_{i}\right)+\sum_{j=1}^{p_{-}} I_{j}^{\sigma}\left(u\left(\sigma_{j}\right)\right) v\left(\sigma_{j}\right)=\int_{0}^{\pi} f(x) \sin n x d x .
$$

Now it follows from (1.5) and (4.2) that (1.6) must hold true.

2. Sufficiency of (1.6). We use the LSD argument similarly as in the proof of Theorem 3.1. For this purpose we define $I_{\tau}^{*}, I_{\sigma}^{*}, I_{\xi}^{*}: H \rightarrow H$ by

$$
\begin{aligned}
\left(I_{\tau}^{*}(u), v\right) & =\sum_{i=1}^{p_{+}} I_{i}^{\tau}\left(u\left(\tau_{i}\right)\right) v\left(\tau_{i}\right), \\
\left(I_{\sigma}^{*}(u), v\right) & =\sum_{j=1}^{p_{-}} I_{j}^{\sigma}\left(u\left(\sigma_{j}\right)\right) v\left(\sigma_{j}\right), \\
\left(I_{\xi}^{*}(u), v\right) & =\sum_{k=1}^{p_{0}} I_{k}^{\xi}\left(u\left(\xi_{k}\right)\right) v\left(\xi_{k}\right) .
\end{aligned}
$$


Problem with impulses $(1.1),(1.4)$ is then equivalent to the operator equation

$$
J u-n^{2} S u+I_{\tau}^{*}(u)+I_{\sigma}^{*}(u)+I_{\xi}^{*}(u)=f^{*} .
$$

Hence, our goal is to prove

$$
\operatorname{deg}\left(J-n^{2} S+I_{\tau}^{*}+I_{\sigma}^{*}+I_{\xi}^{*}-f^{*} ; B_{R}, 0\right) \neq 0
$$

for some $R>0$. To this end we introduce homotopy

$$
\mathcal{H}(\kappa, u):=J u-n^{2} S u-(1-\kappa) \delta S u+\kappa I_{\tau}^{*}(u)+\kappa I_{\sigma}^{*}(u)+\kappa I_{\xi}^{*}(u)-\kappa f^{*},
$$

where $\kappa \in[0,1]$ is a homotopy parameter and $0<\delta<2 n+1$. We prove that there exists $R>0$ such that

$$
\mathcal{H}(\kappa, u) \neq 0
$$

holds for all $u \in H,\|u\|=R$, and all $\kappa \in[0,1]$. The result then follows from (4.4) and from the homotopy invariance of the LSD as in the proof of Theorem 3.1.

We prove (4.4) via contradiction. Let $u_{m} \in H,\left\|u_{m}\right\| \rightarrow \infty, \kappa_{m} \in[0,1]$ be such that $\mathcal{H}\left(\kappa_{m}, u_{m}\right)=0$. Then, setting $v_{m}:=u_{m} /\left\|u_{m}\right\|$, this is equivalent to

$$
\begin{aligned}
J v_{m}-n^{2} S v_{m}-\left(1-\kappa_{m}\right) \delta S v_{m} & +\kappa_{m} \frac{I_{\tau}^{*}\left(u_{m}\right)}{\left\|u_{m}\right\|} \\
& +\kappa_{m} \frac{I_{\sigma}^{*}\left(u_{m}\right)}{\left\|u_{m}\right\|}+\kappa_{m} \frac{I_{\xi}^{*}\left(u_{m}\right)}{\left\|u_{m}\right\|}-\kappa_{m} \frac{f^{*}}{\left\|u_{m}\right\|}=0 .
\end{aligned}
$$

Clearly, $\kappa_{m} f^{*} /\left\|u_{m}\right\| \rightarrow 0$, and also $\kappa_{m} I_{\tau}^{*}\left(u_{m}\right) /\left\|u_{m}\right\| \rightarrow 0, \kappa_{m} I_{\sigma}^{*}\left(u_{m}\right) /\left\|u_{m}\right\| \rightarrow 0$ and $\kappa_{m} I_{\xi}^{*}\left(u_{m}\right) /\left\|u_{m}\right\| \rightarrow 0$ due to the fact that all $I_{i}^{\tau}, I_{j}^{\sigma}$ and $I_{k}^{\xi}$ satisfy (1.3). Similarly as in the proof of Theorem 3.1 we arrive at the limit equation

$$
J v-\left(n^{2}+(1-\kappa) \delta\right) S v=0
$$

with $v \in H,\|v\|=1, \kappa \in[0,1]$. Since $n^{2}+(1-\kappa) \delta<(n+1)^{2}$ due to the choice of $\delta$, (4.5) may occur only if $\kappa=1$ and $v(x)= \pm(1 / n) \sqrt{2 / \pi} \sin n x$. Let us assume first that $v(x)=(1 / n) \sqrt{2 / \pi} \sin n x$. Taking the inner product of $\mathcal{H}\left(\kappa_{m}, u_{m}\right)=0$ with $\sin n x$, we get

$$
\begin{aligned}
\left(1-\kappa_{m}\right) \delta \int_{0}^{\pi} u_{m}(x) \sin n x d x+\kappa_{m} \sum_{i=1}^{p_{+}} I_{i}^{\tau}\left(u_{m}\left(\tau_{i}\right)\right) \sin n \tau_{i} \\
+\kappa_{m} \sum_{j=1}^{p_{-}} I_{j}^{\sigma}\left(u_{m}\left(\sigma_{j}\right)\right) \sin n \sigma_{j}+\kappa_{m} \sum_{k=1}^{p_{0}} I_{k}^{\xi}\left(u_{m}\left(\xi_{k}\right)\right) \sin n \xi_{k} \\
-\kappa_{m} \int_{0}^{\pi} f(x) \sin n x d x=0 .
\end{aligned}
$$


Notice that $\sin n \xi_{k}=0, k=1, \ldots, p_{0}$. Since $v_{m}(x) \rightrightarrows(1 / n) \sqrt{2 / \pi} \sin n x$ (uniformly) on $[0, \pi]$ (by the embedding $H \hookrightarrow C[0, \pi]$ ), we have

$$
\int_{0}^{\pi} u_{m}(x) \sin n x d x>0
$$

from $m \gg 1$. It follows from (4.6) and (4.7) that

$$
\sum_{i=1}^{p_{+}} I_{i}^{\tau}\left(u_{m}\left(\tau_{i}\right)\right) \sin n \tau_{i}+\sum_{j=1}^{p_{-}} I_{j}^{\sigma}\left(u_{m}\left(\sigma_{j}\right)\right) \sin n \sigma_{j} \leq \int_{0}^{\pi} f(x) \sin n x d x .
$$

Since $\sin n \tau_{i}>0, \sin n \sigma_{j}<0$, passing to the limit for $m \rightarrow \infty$ in (4.8), we get

$$
\sum_{i=1}^{p_{+}} I_{i}^{\tau}(+\infty) \sin n \tau_{i}+\sum_{j=1}^{p_{-}} I_{j}^{\sigma}(-\infty) \sin n \sigma_{j} \leq \int_{0}^{\pi} f(x) \sin n x d x
$$

a contradiction with the second inequality in (1.6).

If $v(x)=-(1 / n) \sqrt{2 / \pi} \sin n x$ we derive similarly a contradiction with the first inequality in (1.6).

Theorem 4.2. Let $\lambda=n^{2}$ for $n \in \mathbb{N}, p_{+}+p_{-}=0$, and $I_{k}^{\xi}: \mathbb{R} \rightarrow \mathbb{R}$ be continuous functions, $k=1, \ldots, p_{0}$ (not necessarily sublinear). Then (1.1), (1.4) has a solution if and only if $f \in L^{2}(0, \pi)$ satisfies

$$
\int_{0}^{\pi} f(x) \sin n x d x=0 .
$$

Proof. 1. Necessity of (4.9). Let $u$ be a solution of (1.1), (1.4). Then

$\int_{0}^{\pi} u^{\prime}(x) v^{\prime}(x) d x-n^{2} \int_{0}^{\pi} u(x) v(x) d x+\sum_{k=1}^{p_{0}} I_{k}^{\xi}\left(u\left(\xi_{k}\right)\right) v\left(\xi_{k}\right)=\int_{0}^{\pi} f(x) \sin n x d x$.

Choose $v(x)=\sin n x$, an eigenfunction associated with $n^{2}$, and use the fact $\sin n \xi_{k}=0, k=1, \ldots, p_{0}$, to derive (4.9).

2. Sufficiency of (4.9). Elementary calculation (variation of constants formula) yields that under condition (4.9) the function

$$
w(x)=A \sin n x+\frac{1}{n} \int_{0}^{x} f(\sigma) \cos n \sigma d \sigma \cdot \sin n x-\frac{1}{n} \int_{0}^{x} f(\sigma) \sin n \sigma d \sigma \cdot \cos n x,
$$

$A \in \mathbb{R}$, is a (general) solution of the equation $-u^{\prime \prime}-n^{2} u=f$ which satisfies boundary conditions $u(0)=u(\pi)=0$. Set

$$
u_{k}(x)=c_{k} \sin n x+\frac{1}{n} \int_{0}^{x} f(\sigma) \cos n \sigma d \sigma \cdot \sin n x-\frac{1}{n} \int_{0}^{x} f(\sigma) \sin n \sigma d \sigma \cdot \cos n x,
$$

$x \in \mathcal{I}_{k}, \mathcal{I}_{k}=\left(\xi_{k}, \xi_{k+1}\right), k=0, \ldots, p_{0}$, where $\xi_{0}=0, \xi_{p_{0}+1}=\pi$. Then $\Delta u^{\prime}\left(\xi_{k}\right)=$ $I_{k}^{\xi}\left(u\left(\xi_{k}\right)\right)$ if and only if we choose

$$
c_{k}-c_{k+1}=\frac{1}{n} I_{k}^{\xi}\left(\frac{\cos n \xi_{k}}{n} \int_{0}^{\xi_{k}} f(\sigma) \sin n \sigma d \sigma\right), \quad k=0, \ldots, p_{0} .
$$


The solution of $(1.1),(1.4)$ then consists of functions $u_{k}, k=1, \ldots, p_{0}$, with the choice of constants $c_{k}$ given by (4.10).

Note that we have an analytic expression of the solution. It follows from (4.10) that the solution is not determined uniquely. The constant $c_{0} \in \mathbb{R}$ can be chosen arbitrarily, the values of $c_{k}, k=1, \ldots, p_{0}$, then follow from recurrent formula (4.10).

\section{Combined effect of restoring force and impulses}

Weak and strong solutions of (1.14), (1.4) are defined with obvious modifications similarly as weak and strong solutions of (1.1), (1.2) in Section 2. We introduce an operator $G: H \rightarrow H$ by

$$
(G(u), v)=\int_{0}^{\pi} g(u(x)) v(x) d x, \quad u, v \in H .
$$

Then $G$ is a (nonlinear) compact operator due to the compact embedding $H \hookrightarrow$ $C[0, \pi]$ and the continuity of the Nemyt'skiu operator $g(\cdot): C[0, \pi] \rightarrow C[0, \pi]$ (see $[5$, Chapter 3] for details). Problem with impulses $(1.14),(1.2)$ is then equivalent to the operator equation

$$
J u-\lambda S u+G(u)+I^{*}(u)=f^{*} .
$$

Similarly, problem with impulses (1.14), (1.4) is equivalent to

$$
J u-\lambda S u+G(u)+I_{\tau}^{*}(u)+I_{\sigma}^{*}(u)+I_{\xi}^{*}(u)=f^{*} .
$$

Using the sublinear growth of $g$ at $\pm \infty$, obvious modifications of the proof of Theorem 3.1 yield the nonresonance result.

Theorem 5.1. Let $\lambda \neq n^{2}, n \in \mathbb{N}, g$ and $I_{j}, j=1, \ldots, p$, be as in Section 1 . Then (1.14), (1.2) has a solution for arbitrary $f \in L^{2}(0, \pi)$.

ProOF. We use the properties of the LSD and consider the homotopy

$$
\mathcal{H}(\kappa, u):=J u-\lambda S u-(1-\kappa) \delta S u+\kappa G(u)+\kappa I^{*}(u)-\kappa f^{*},
$$

$\kappa \in[0,1], \delta>0$ such that $\lambda+(1-\kappa) \delta \neq n^{2}, n \in \mathbb{N}$, for any $\kappa \in[0,1]$. Homotopy invariance of the LSD then yields the existence of a solution of (5.1).

As for the resonance case, we have the following assertion.

TheOREM 5.2. Let $\lambda=n^{2}$ for some $n \in \mathbb{N}, g, \tau_{i}, \sigma_{j}, \xi_{k}, I_{i}^{\tau}, I_{j}^{\sigma}, I_{k}^{\xi}$ be as in Section 1. Then (1.14), (1.4) has a solution if and only if $f \in L^{2}(0, \pi)$ satisfies (1.16). 
Proof. 1. Necessity of (1.16). Similarly as in the proof of Theorem 4.1, we arrive at

$$
\begin{aligned}
\int_{0}^{\pi} g(u(x)) \sin n x d x+\sum_{i=1}^{p_{+}} I_{i}^{\tau}\left(u\left(\tau_{i}\right)\right) \sin n \tau_{i}+\sum_{j=1}^{p_{-}} I_{j}^{\sigma}\left(u\left(\sigma_{j}\right)\right) \sin n \sigma_{j} \\
\quad=\int_{0}^{\pi} f(x) \sin n x d x .
\end{aligned}
$$

Condition (1.16) then immediately follows from (1.13) and (1.5).

2. Sufficiency of (1.16). Similarly as in the proof of the Theorem 4.1, our goal is to prove that

$$
\operatorname{deg}\left(J-n^{2} S+G+I_{\tau}^{*}+I_{\sigma}^{*}+I_{\xi}^{*}-f^{*} ; B_{R}, 0\right) \neq 0
$$

for some $R>0$. To this end we introduce homotopy

$\mathcal{H}(\kappa, u):=J u-n^{2} S u-(1-\kappa) \delta S u+\kappa G(u)+\kappa I_{\tau}^{*}(u)+\kappa I_{\sigma}^{*}(u)+\kappa I_{\xi}^{*}(u)-\kappa f^{*}$

with homotopy parameter $\kappa \in[0,1]$ and $0<\delta<2 n+1$. A contradiction argument leads to the same limit equation (4.5). If $v(x)=(1 / n) \sqrt{2 / \pi} \sin n x$, we take the inner product of $\mathcal{H}\left(\kappa_{m}, u_{m}\right)=0$ with $\sin n x$ and get

$$
\begin{aligned}
& \left(1-\kappa_{m}\right) \delta \int_{0}^{\pi} u_{m}(x) \sin n x d x+\kappa_{m} \int_{0}^{\pi} g\left(u_{m}(x)\right) \sin n x d x \\
& +\kappa_{m} \sum_{i=1}^{p_{+}} I_{i}^{\tau}\left(u_{m}\left(\tau_{i}\right)\right) \sin n \tau_{i}+\kappa_{m} \sum_{j=1}^{p_{-}} I_{j}^{\sigma}\left(u_{m}\left(\sigma_{j}\right)\right) \sin n \sigma_{j} \\
& \quad+\kappa_{m} \sum_{k=1}^{p_{0}} I_{k}^{\xi}\left(u_{m}\left(\xi_{k}\right)\right) \sin n \xi_{k}-\kappa_{m} \int_{0}^{\pi} f(x) \sin n x d x=0 .
\end{aligned}
$$

Using (4.7), we arrive at

$$
\begin{array}{r}
\int_{0}^{\pi} g\left(u_{m}(x)\right) \sin n x d x+\sum_{i=1}^{p_{+}} I_{i}^{\tau}\left(u_{m}\left(\tau_{i}\right)\right) \sin n \tau_{i}+\sum_{j=1}^{p_{-}} I_{j}^{\sigma}\left(u_{m}\left(\sigma_{j}\right)\right) \sin n \sigma_{j} \\
\leq \int_{0}^{\pi} f(x) \sin n x d x .
\end{array}
$$

Passing to the limit for $m \rightarrow \infty$ we obtain from here

$$
\begin{aligned}
g(+\infty) \int_{0}^{\pi}(\sin n x)^{+} d x-g(-\infty) & \int_{0}^{\pi}(\sin n x)^{-} d x+\sum_{i=1}^{p_{+}} I_{i}^{\tau}(+\infty) \sin n \tau_{i} \\
& +\sum_{j=1}^{p_{-}} I_{j}^{\sigma}(-\infty) \sin n \sigma_{j} \leq \int_{0}^{\pi} f(x) \sin n x d x,
\end{aligned}
$$

a contradiction with the second inequality in (1.16). 
Similarly, we arrive at a contradiction with the first inequality in (1.16) in the case $v(x)=-(1 / n) \sqrt{2 / \pi} \sin n x$. The homotopy invariance of the LSD then yields (5.3) and the proof is finished.

\section{REFERENCES}

[1] R.P. Agarwal, D. Franco and D. O'Regan, Singular boundary value problems for first and second order impulsive differential equations, Aequationes Math. 69 (2005), 83-96.

[2] T.E. CARTER, Optimal impulsive space trajectories based on linear equations, J. Optim. Theory Appl. 70 (1991), 277-297.

[3] _ Necessary and sufficient conditions for optimal impulsive rendezvous with linear equations of motion, Dynam. Control 10 (2000), 219-227.

[4] P. DrÁBEK, Solvability and Bifurcations of Nonlinear Equations, Longman Scientific and Technical, Pitman Res. Notes in Math. Series 264, Longman 1992.

[5] P. Drábek and J. Milota, Methods of Nonlinear Analysis, Applications to Differential Equations, Second Edition, Birkhäuser, Springer, Basel, 2013.

[6] E.M. LAndesman And A.C. LAZER, Nonlinear perturbations of linear elliptic boundary value problems at resonance, J. Math. Mech. 19 (1970), 609-623.

[7] X. Lin AND D. JiAng, Multiple positive solutions of Dirichlet boundary value problems for second order impulsive differential equations, J. Math. Anal. Appl. 321 (2006), 501-514.

[8] X. Liu AND A.R. Willms, Impulsive controllability of linear dynamical systems with applications to maneuvers of Spacecraft, Math. Probl. Eng. 2 (1996), 277-299.

[9] J.J. Nieto And D. O'Regan, Variational approach to impulsive differential equations, Nonlinear Anal. Real World Appl. 10 (2009), 680-690.

[10] A.F.B.A. Prado, Bi-impulsive control to build a satellite constellation, Nonlinear Dyn. Syst. Theory 5 (2005), 169-175.

[11] D. QIAn AND X. LI, Periodic solutions for ordinary differential equations with sublinear impulsive effects, J. Math. Anal. Appl. 303 (2005), 288-303.

[12] I. RACHŮNKOVÁ AND M. TVRDÝ, Existence results for impulsive second-order periodic problems, Nonlinear Anal. 59 (2004), 133-146.

[13] Y. Tian And W. Ge, Applications of variational methods to boundary value problem for impulsive differential equations, Proc. Edinb. Math. Soc. 51 (2008), 509-517.

PAVEL DrÁBEK

Department of Mathematics and NTIS

University of West Bohemia

Plzeň, CZECH REPUBLIC

E-mail address: pdrabek@kma.zcu.cz

Martina Langerová

Department of Mathematics

University of Žilina

Žilina, SLOVAKIA

and

NTIS, University of West Bohemia

Plzeň, CZECH REPUBLIC

E-mail address: mlanger@ntis.zcu.cz

TMNA : Volume $44-2014-\mathrm{N}^{\mathrm{O}} 1$ 